\title{
Competing for Customers' Attention: Advertising When Consumers Have Imperfect Memory
}

\author{
Oksana Loginova \\ University of Missouri-Columbia \\ Department of Economics \\ 118 Professional Bldg \\ Columbia, MO 65211 \\ loginovao@missouri.edu
}

December 2006

\begin{abstract}
This paper applies the theory of memory for advertising, developed in the consumer behavior literature, to an industrial organization setting to provide insight into advertising strategies in imperfectly competitive markets. There are two firms and infinitely many identical consumers. The firms produce a homogeneous product and distribute their brands through a common retailer. Consumers randomly arrive and are willing to buy one unit of the product. They are unaware of the existence of a particular brand unless they remember an ad describing it. Under "retroactive interference" consumers remember recently seen ads and forget about ads they saw in the past. Under "proactive interference" the ability of consumers to recall new ads is hampered by past ad exposure. The equilibrium of the advertising game is characterized for both proactive and retroactive interferences across three strategic settings. In the Simultaneous Move setting, the firms' equilibrium advertising frequencies, remarkably, do not depend on the type of interference. In the Sequential Move and Dynamic settings, proactive and retroactive interferences do give rise to different equilibrium outcomes.
\end{abstract}

JEL Classifications: C73, D11, D43, L13, M37.

Keywords: advertising, memory, forgetting, competitive interference. 


\section{Introduction}

Traditionally, advertising has been studied by two very different subfields of social science, economics and psychology ${ }^{1}$. The economic literature on advertising has been concerned with such important questions as the informational content of ads, the socially optimal level of advertising, and firm rivalry. The psychology literature has (among other things) dealt with memory. In this paper, a model is constructed that incorporates economics and psychology to examine the interplay between advertising and memory with respect to firms' advertising decisions.

The branch of economic literature to which the model presented in this paper most closely concerns is informative advertising in new markets, where consumers are a priori uninformed about the existence of goods ${ }^{2}$. In a pioneering study, Butters (1977) investigated the setting in which firms sell a homogeneous product, and consumers learn about product availability and prices through advertising. He showed that the resulting equilibrium involves price dispersion, with high prices advertised more intensively than low prices. More striking was his finding that free markets produce the socially optimal level of advertising. Stegman (1991) modified Butters' model to allow buyers to have heterogeneous reservation values. He showed that each firm under-advertises in equilibrium. Grossman and Shapiro (1984) examined informative advertising with spatially differentiated products, finding that the market-determined levels of advertising are excessive. The current paper takes a very different tack from the extant literature on informative advertising. Specifically, in this paper a setting is studied in which consumers occasionally forget about the availability of goods and firms advertise in order to "remind" them.

The psychology literature on advertising is also vast and rich. Of particular interest from the perspective of this study are the theories concerning why consumers might forget the ads they have seen. Most widely known among them are decay theory, interference theory,

\footnotetext{
${ }^{1}$ Of course, advertising is one of the primary subjects studied in the field of marketing. Most papers in marketing concerning advertising, however, can be classified as having either economic or psychologic foundations.

${ }^{2}$ For informative advertising in established markets, where consumers are well informed about availability and properties of goods, see, for example, Bester and Petrakis (1995), LeBlanc (1998).
} 
and situational forgetting (Tulving and Pearlston 1966) ${ }^{3}$. According to the decay theory, memory traces are formed when a person learns information, and when no longer in use, these traces gradually fade with the passage of time (Ebbinghaus 1885, Woodworth 1938). The main argument against this intuitively understandable theory is that it does not account for processes occurring between initial learning and retrieval.

Alternatively, forgetting may occur because in the limited storage capacity earlier learning competes, or interferes, with later learning (Jenkins and Dallenbach 1924, Underwood 1957, Murnane and Shiffrin 1991). The interference theory can work either way. Under proactive interference, earlier learning makes it more difficult to encode new information. Under retroactive interference, later learning hampers the ability to remember previously learned information.

Another theory from the information processing approach was introduced by Tulving (1974), who suggested that people forget certain information because the features of current information do not match those they try to retrieve. The theory of situational forgetting implies that the traces of old information are not lost but require adequate stimulation for their activation and retrieval.

These theories of forgetting, the competitive interference phenomenon in particular, have been carried over into consumer behavior literature in analysis of consumer memory for advertising. Burke and Srull (1988) were the first to conduct a series of experiments that examine interference-induced forgetting in an advertising context. The first experiment tested how consumer memory for magazine ads is affected by subsequent exposure to competitors' ads, finding that recall is significantly diminished by higher level of retroactive interference. The second experiment examined how the learning of new ads is affected by past ad exposure, demonstrating that proactive interference has a negative effect on consumer ability to learn and retain new information presented in future advertising. The third experiment investigated how consumer memory for a particular ad is affected by the ad's level of repetition in various competitive environments. The results suggested that repetition of the same ad only has a positive effect on recall of the advertised product when there is little or no advertising for

\footnotetext{
${ }^{3}$ See Solso (1988) for a textbook review for these theories.
} 
competitive products. Therefore, higher levels of ad repetition may not overcome detrimental effects of ad interference ${ }^{4}$.

In subsequent studies, researchers explored the role of brand familiarity in competitive advertising. When consumers are exposed to an ad for a familiar and mature brand, the ad is easily recognized and stored in memory in the well-established schema for the brand. Kent and Allen (1994) showed experimentally that, in comparison to unfamiliar brands, advertising information related to familiar brands is less sensitive to competitive interference ${ }^{5}$.

Summarizing the above, economists have dealt with advertising in competitive markets without taking into account the role of memory. Psychologists have studied consumer memory for advertising but not, of course, equilibrium advertising strategies employed by firms. This paper brings the two together. Its purpose is to construct a model of consumer memory for advertising, based on the decay and interference theories of forgetting, and to embed this model in a setting of oligopolistic firm rivalry.

The agents of the model presented in this paper are two firms and infinitely many identical consumers of total mass one. The firms produce a homogeneous good; firm $A$ produces brand $A$ and firm $B$ produces brand $B$. The firms distribute their brands through a common retailer. Consumers randomly arrive at the retailer and are willing to buy one unit of the good. To provide a role for advertising, it is assumed that a consumer is unaware of the existence of a particular brand unless she saw and remember an ad describing it. All the events - arrivals to the retailer, advertising messages, and forgetting - are Poisson processes.

Consumer memory for advertising is modelled as a continuous-time Markov chain that takes values in the state space consisting of three elements: "remember an ad describing brand $A$ ", "remember an ad describing brand $B$ ", and "unaware of the product existence" 6 . Initially, all consumers are in state "unaware of the product existence". A consumer's memory jumps to state "remember an ad describing brand $A$ " or "remember an ad describing brand $B$ " once she sees an advertising message of the respective firm. Suppose the consumer saw firm $A$ 's

\footnotetext{
${ }^{4}$ See also Keller $(1987,1991)$.

${ }^{5}$ See also Jewell and Unnava (2003), Kumar and Krishnan (2004).

${ }^{6}$ In Wilson (2002), a decision-maker is also restricted to a finite set of memory states. The paper explores how the decision-maker with a bounded memory updates her beliefs upon receiving new information, finding that the agent displays a confirmatory and overconfidence/underconfidence biases.
} 
ad first. If the consumer subsequently sees firm $B$ 's ad, then, under retroactive interference, the memory process jumps to state "remember an ad describing brand $B$ ", as new information facilitates forgetting of what was learned previously. Under proactive interference, the memory process stays in state "remember an ad describing brand $A$ ", as previous learning hampers the ability to encode new information. Finally, the consumer may simply forget about the brand she is aware of, and return to state "unaware of the product existence", a manifestation of the decay theory.

The firms set their prices equal to the consumer valuation for the good and compete in advertising frequencies. The equilibrium outcome of the advertising game is characterized for both proactive and retroactive interferences across three settings: simultaneous, sequential, and dynamic moves. In the Simultaneous Move setting, the firms make their advertising decisions simultaneously at the beginning of the game. In this setup, the equilibrium advertising frequencies, remarkably, do not depend on the type of interference. It is shown that in this setting each firm over-advertises in equilibrium relative to the social optimum.

In the Sequential Move setting, where one firm makes its advertising decision at the beginning of the game, and the other firm enters the market and chooses its advertising frequency at some (exogenously given) time $T$, proactive and retroactive interferences give rise to different equilibrium outcomes. Results show that, in order to undermine the advertising incentives of the second firm, the first firm chooses higher advertising frequency under retroactive interference than under proactive. In the third setting studied, the firms choose their advertising frequencies twice, at the beginning of the game, and then at time $T$. As in the Sequential Move setting, the equilibrium outcome in the Dynamic setting depends on the interference type. At the beginning of the game, when competing for consumers, the firms choose higher advertising frequencies under proactive interference than under retroactive interference.

In the next section, the formal model is presented. The Simultaneous Move setting is analyzed in Section 3. Some technical lemmas are contained in Section 4. In Sections 5 and 6, the Sequential Move and Dynamic settings are explored respectively. Concluding remarks appear in Section 7. All proofs are relegated to an Appendix. 


\section{The Model}

\section{Supply Side}

On the supply side, there are two firms, $A$ and $B$, that produce a homogeneous product at zero marginal cost. The firms distribute their brands through a common retailer.

\section{Demand Side}

The demand side consists of infinitely many identical consumers of total mass 1 . Consumers are risk-neutral, possess continuous-time discount rate $r>0$, and have a sequence of unit demands for the product. In particular, a consumer receives a gross payoff of $v=1$ from consuming the product whenever she (i) visits the retailer (i.e., goes shopping) and (ii) remembers to buy the product.

Let $N_{t}$ denote the number of times a consumer visits the retailer by time $t . N_{t}$ is a Poisson process with rate parameter $\sigma>0$. That is,

(i) The number of visits during one time interval is independent of the number of visits during a different (non-overlapping) time interval;

(ii) In a small time interval $\Delta t$ the consumer visits the retailer with probability about $\sigma \Delta t$,

$$
\operatorname{Pr}\left\{N_{t+\Delta t}=N_{t}+1\right\}=\sigma \Delta t+o(\Delta t)
$$

Here $o(\Delta t)$ is much smaller than $\Delta t$, i.e.,

$$
\lim _{\Delta t \rightarrow 0} \frac{o(\Delta t)}{\Delta t}=0^{7}
$$

\section{Advertising Technology}

To provide the role for advertising, it is assumed that consumers are unaware of the existence of a particular brand unless they remember an ad describing it. The firms decide on advertising

\footnotetext{
${ }^{7}$ For the definition of a Poisson Process see, for example, Doob (1953), pp. 398-404, or Lawler (1995), pp. $53-56$
} 
frequencies $\alpha_{A}$ and $\alpha_{B}$ - the rate parameters of Poisson processes that advertising messages follow. Each consumer receives messages independently of other consumers.

The (discounted) cost needed to achieve advertising frequency $\alpha \geq 0$ is $c(\alpha) / r$, where $c(\cdot)$ is a convex and increasing function, $c^{\prime}>0, c^{\prime \prime}>0$. The boundary assumptions, $c(0)=c^{\prime}(0)=0$ and $\lim _{\alpha \rightarrow \infty} c^{\prime}(\alpha)=\infty$, guarantee existence of an interior solution.

\section{Consumer Memory for Advertising}

Consumer memory for advertising is a continuous-time Markov process $M_{t}$ taking values in the state space consisting of three elements: $s_{\varnothing}, s_{A}$ and $s_{B}$. A consumer remembers an ad describing brand $A$ (brand $B$ ) if her memory is in state $s_{A}$ (state $s_{B}$ ). The consumer is unaware of the product existence if her memory is in state $s_{\varnothing}$. It follows that consumers never remember two ads describing each brand at a time. In other words, memory has a limited storage capacity.

The probability that the memory process in state $s_{\varnothing}$ jumps to state $s_{A}$ (state $s_{B}$ ) in a small time interval of length $\Delta t$ is equal to the probability that the consumer sees firm $A$ 's (firm $B$ 's) advertising message:

$$
\begin{aligned}
& \operatorname{Pr}\left\{M_{t+\Delta t}=s_{A} \mid M_{t}=s_{\varnothing}\right\}=\alpha_{A} \Delta t+o(\Delta t), \\
& \operatorname{Pr}\left\{M_{t+\Delta t}=s_{B} \mid M_{t}=s_{\varnothing}\right\}=\alpha_{B} \Delta t+o(\Delta t) .
\end{aligned}
$$

Conversely, the probability that the process in state $s_{A}$ (state $s_{B}$ ) jumps to state $s_{\varnothing}$ is proportional to the rate of forgetting $\phi$ :

$$
\begin{aligned}
& \operatorname{Pr}\left\{M_{t+\Delta t}=s_{\varnothing} \mid M_{t}=s_{A}\right\}=\phi \Delta t+o(\Delta t), \\
& \operatorname{Pr}\left\{M_{t+\Delta t}=s_{\varnothing} \mid M_{t}=s_{B}\right\}=\phi \Delta t+o(\Delta t) .
\end{aligned}
$$

Parameter $\phi \geq 0$ reflects the general observation that people forget as time passes (i.e., the decay theory).

Next, the probability that the process in state $s_{A}$ jumps to state $s_{B}$ is negligibly small 
Figure 1: Transition Diagrams
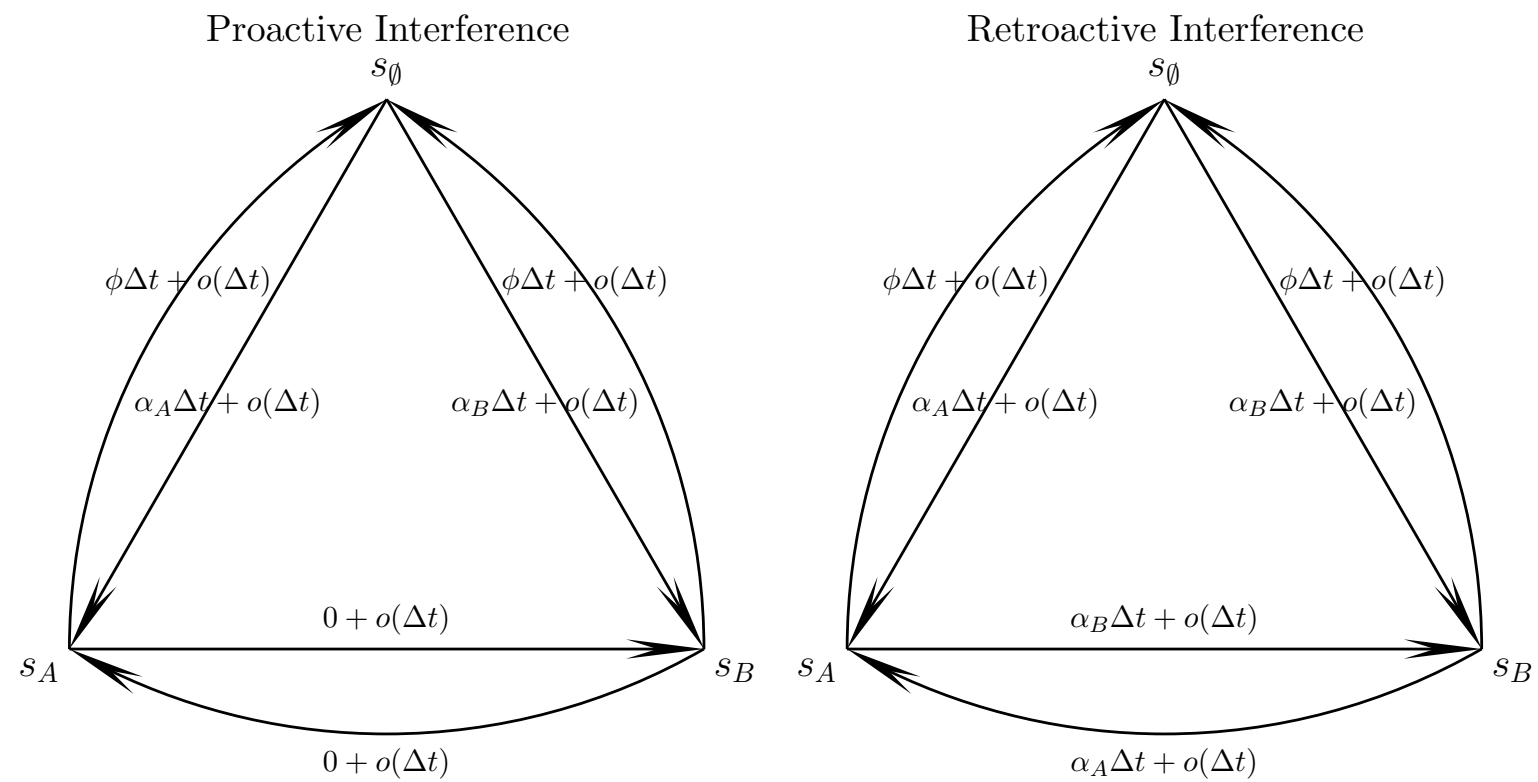

in case of proactive memory: old information hampers the ability to learn new information. This probability is proportional to $\alpha_{B}$ in case of retroactive memory: new information "pushes out" old information. That is,

$$
\operatorname{Pr}\left\{M_{t+\Delta t}=s_{B} \mid M_{t}=s_{A}\right\}= \begin{cases}o(\Delta t), & \text { if memory is proactive } \\ \alpha_{B} \Delta t+o(\Delta t), & \text { if memory is retroactive }\end{cases}
$$

Similarly,

$$
\operatorname{Pr}\left\{M_{t+\Delta t}=s_{A} \mid M_{t}=s_{B}\right\}= \begin{cases}o(\Delta t), & \text { if memory is proactive } \\ \alpha_{A} \Delta t+o(\Delta t), & \text { if memory is retroactive }\end{cases}
$$

It is assumed $M_{0}=s_{\varnothing}$. That is, at $t=0$ consumers are unaware about the existence of the product. Figure 1 shows graphically the probabilities of transition from one state to another for the two memory types. 


\section{Equilibrium Concept}

There is no price competition between the firms, as, by assumption, consumers never remember two ads describing each brand at a time. The firms set their prices equal to 1, the consumers' valuation for the good.

The strategic variables of the firms are advertising frequencies. The equilibrium concept employed is subgame perfect Nash equilibrium. In the Simultaneous Move setting, the firms choose their advertising frequencies simultaneously. An equilibrium is a pair $\left(\alpha_{A}, \alpha_{B}\right)$.

In the Sequential Move setting, firm $A$ chooses its advertising frequency first; firm $B$ observes firm $A$ 's frequency, then chooses its own at exogenous time $T>0$. In this case, an equilibrium is a pair $\left(\alpha_{A}, \alpha_{B}(\cdot)\right)$.

In the Dynamic setting, the firms choose their advertising frequencies twice: at $t=0$, and then at $t=T$. Therefore, an equilibrium is a quadruple $\left(\alpha_{A 0}, \alpha_{A T}(\cdot, \cdot) ; \alpha_{B 0}, \alpha_{B T}(\cdot, \cdot)\right)$.

\section{$3 \quad$ Simultaneous Move Setting}

In this section the setting in which firm $A$ and firm $B$ make their advertising decisions simultaneously at the beginning of the game is analyzed.

Let $p_{s}(t)$ denote the probability that the memory process is in state $s$ at time $t$ :

$$
p_{s}(t) \equiv \operatorname{Pr}\left\{M_{t}=s\right\}
$$

where the dependence on $\alpha_{A}$ and $\alpha_{B}$ is suppressed for the moment.

From the aggregate point of view, $p_{s_{A}}(t)$ and $p_{s_{B}}(t)$ are the fractions of consumers that at time $t$ remember ads describing brands $A$ and $B$ respectively, while $p_{s_{\varnothing}}(t)$ is the fraction of consumers that are unaware of the product existence.

When memory is proactive, functions $p_{s_{\varnothing}}(t), p_{s_{A}}(t)$ and $p_{s_{B}}(t)$ satisfy the following system 
of linear differential equations ${ }^{8}$,

$$
\left\{\begin{array}{l}
p_{s_{\varnothing}}^{\prime}=-\left(\alpha_{A}+\alpha_{B}\right) p_{s \varnothing}+\phi\left(p_{s_{A}}+p_{s_{B}}\right), \\
p_{s_{A}}^{\prime}=-\phi p_{s_{A}}+\alpha_{A} p_{s_{\varnothing}} \\
p_{s_{B}}^{\prime}=-\phi p_{s_{B}}+\alpha_{B} p_{s_{\varnothing}} .
\end{array}\right.
$$

The first differential equation corresponds to state $s_{\varnothing}$. The change in number of consumers that are unaware of the product existence is on the left hand side of the equation. The minus term on the right hand side is the rate at which consumers "leave" state $s_{\varnothing}, \alpha_{A}+\alpha_{B}$, multiplied by the number of consumers in this state. (A consumer leaves state $s_{\varnothing}$ when she observes an ad of firm $A$ or $B$.) The plus term on the right hand side is the rate at which consumers "arrive at" state $s_{\varnothing}, \phi$, multiplied by the number of consumers in states $s_{A}$ and $s_{B}$. (A consumer arrives at state $s_{\varnothing}$ from state $s_{A}$ or $s_{B}$ when she forgets about the existence of brand $A$ or $B$, respectively.)

The second equation corresponds to state $s_{A}$. The change in number of consumers that know about brand $A$ 's existence is on the left hand side of the equation. The minus term on the right hand side is the rate at which consumers leave state $s_{A}, \phi$, multiplied by the number of consumers in this state. (A consumer leaves state $s_{A}$ when she forgets about the brand.) The plus term on the right hand side is the rate at which consumers arrive at state $s_{A}, \alpha_{A}$, multiplied by the number of consumers in state $s_{\varnothing}$. (A consumer arrives at state $s_{A}$ from state $s_{\varnothing}$ when she observes an ad of firm $A$.) Similar logic applies to the third differential equation that corresponds to state $s_{B}$.

When memory is retroactive,

$$
\left\{\begin{array}{l}
p_{s_{\varnothing}}^{\prime}=-\left(\alpha_{A}+\alpha_{B}\right) p_{s_{\varnothing}}+\phi\left(p_{s_{A}}+p_{s_{B}}\right) \\
p_{s_{A}}^{\prime}=-\left(\alpha_{B}+\phi\right) p_{s_{A}}+\alpha_{A}\left(p_{s_{B}}+p_{s_{\varnothing}}\right) \\
p_{s_{B}}^{\prime}=-\left(\alpha_{A}+\phi\right) p_{s_{B}}+\alpha_{B}\left(p_{s_{A}}+p_{s_{\varnothing}}\right) .
\end{array}\right.
$$

The first differential equation, corresponding to state $s_{\varnothing}$, coincides with its proactive coun-

\footnotetext{
${ }^{8}$ See, for example, Lawler 1995, pp. 56-61.
} 
terpart. The second and third equations are different. On the left hand side of the second equation is the change in number of consumers that know about brand $A$ 's existence. The minus term on the right hand side is the rate at which consumers leave state $s_{A}, \phi+\alpha_{B}$, multiplied by the number of consumers in this state. (Under retroactive interference, a consumer leave state $s_{A}$ when she either forget about the brand or when she observes an ad describing brand $B$.) The plus term on the right hand side is the rate at which consumers arrive at state $s_{A}, \alpha_{A}$, multiplied by the number of consumers in states $s_{\varnothing}$ and $s_{B}$. (A consumer arrives at state $s_{A}$ from state $s_{\varnothing}$ or $s_{B}$ when she observes an ad message of firm $A$.) Similar logic applies to the third differential equation.

It turns out that under initial conditions $p_{s_{\varnothing}}(0)=1, p_{s_{A}}(0)=p_{s_{B}}(0)=0$ (the process is in state $s_{\varnothing}$ at $\left.t=0\right)$, the two systems generate the same solution.

Lemma 1. Probabilities $p_{s}(t), s=s_{\varnothing}, s_{A}$ and $s_{B}$, do not depend on the type of consumer memory and are given by

$$
\begin{aligned}
p_{\text {ø }}\left(t ; \alpha_{A}, \alpha_{B}\right) & =\frac{\phi+\left(\alpha_{A}+\alpha_{B}\right) e^{-\left(\alpha_{A}+\alpha_{B}+\phi\right) t}}{\alpha_{A}+\alpha_{B}+\phi}, \\
p_{s_{i}}\left(t ; \alpha_{A}, \alpha_{B}\right) & =\frac{\alpha_{i}\left(1-e^{-\left(\alpha_{A}+\alpha_{B}+\phi\right) t}\right)}{\alpha_{A}+\alpha_{B}+\phi},
\end{aligned}
$$

where $i=A, B$.

Limiting probabilities $\bar{p}_{s} \equiv \lim _{t \rightarrow \infty} p_{s}(t), s=s_{\varnothing}, s_{A}, s_{B}$, represent long-run behavior of the memory process. It is easy to see that these probabilities are proportional to $\phi, \alpha_{A}$ and $\alpha_{B}$, respectively:

$$
\begin{gathered}
\bar{p}_{s \varnothing}=\frac{\phi}{\alpha_{A}+\alpha_{B}+\phi}, \\
\bar{p}_{s_{i}}=\frac{\alpha_{i}}{\alpha_{A}+\alpha_{B}+\phi},
\end{gathered}
$$

where $i=A, B$.

Lemma 1 allows to compute the (discounted) profits of the firms, as functions of $\alpha_{A}$ and $\alpha_{B}$. In a small time period $[t, t+\Delta t]$, a consumer buys brand $i$ with probability $\sigma \Delta t$ (the 
probability that she visits the retailer) multiplied by $p_{s_{i}}\left(t ; \alpha_{A}, \alpha_{B}\right)$ (the probability that she remembers brand $i$ ). Firm $i$ 's profit function is, therefore,

$$
\Pi_{i}\left(\alpha_{A}, \alpha_{B}\right)=\int_{0}^{\infty} e^{-r t} p_{s_{i}}\left(t ; \alpha_{A}, \alpha_{B}\right) \sigma d t-\frac{c\left(\alpha_{i}\right)}{r} .
$$

Performing simple algebra yields the following proposition.

Proposition 1 (Profit Functions). In the Simultaneous Move setting, the profit functions of the firms do not depend on the type of consumer memory and are given by

$$
\Pi_{i}\left(\alpha_{A}, \alpha_{B}\right)=\frac{\sigma \alpha_{i}}{r\left(\alpha_{A}+\alpha_{B}+r+\phi\right)}-\frac{c\left(\alpha_{i}\right)}{r}
$$

where $i=A, B$.

In line with economic intuition, the gross profit of firm $i$ (the first term of $\Pi_{i}$ ) is increasing in its own advertising frequency, decreasing in the advertising frequency of its competitor, and is proportional to the shopping rate.

Since probabilities $p_{s}\left(t ; \alpha_{A}, \alpha_{B}\right), s=s_{A}, s_{B}$, are the same for the two types of consumer memory, the profit functions are the same. This result is due to symmetry in the initial conditions - at $t=0$ consumers do not know brand $A$, nor brand $B$.

Proposition 2 (Over-Advertising in Competitive Equilibrium). In the Simultaneous Move setting:

(i) The social optimum is $\alpha_{A}^{\dagger}=\alpha_{B}^{\dagger}=\alpha^{\dagger}$, where $\alpha^{\dagger}$ is implicitly defined by

$$
\frac{\sigma(r+\phi)}{\left(2 \alpha^{\dagger}+r+\phi\right)^{2}}=c^{\prime}\left(\alpha^{\dagger}\right)
$$

(ii) Equilibrium is unique and symmetric, $\alpha_{A}^{*}=\alpha_{B}^{*}=\alpha^{*}$, where $\alpha^{*}$ is implicitly defined by

$$
\frac{\sigma\left(\alpha^{*}+r+\phi\right)}{\left(2 \alpha^{*}+r+\phi\right)^{2}}=c^{\prime}\left(\alpha^{*}\right)
$$

(iii) Advertising is socially excessive, $\alpha^{*}>\alpha^{\dagger}$. 
While a social planner would aim to increase the fraction of consumers that know about the product availability through the firms' advertising messages, each firm cares only about the fraction of consumers that remember its own brand. As a result, the two firms over-advertise in equilibrium.

In the simple setting where the firms choose their advertising frequencies simultaneously at the beginning of the game, the equilibrium outcome is the same independent of whether consumer memory is proactive or retroactive. However, if some sort of asymmetry is introduced (for example, one firm enters the market and chooses its advertising frequency first, as in the Sequential Move setting), or if the firms make their advertising decisions dynamically (the Dynamic setting), the equilibrium outcome does depend on the type of memory process.

For the rest of the paper $\phi=0$ is assumed. That is, no direct forgetting occurs. This assumption greatly simplifies the analysis and makes the difference between proactive and retroactive interference as stark as possible.

\section{Technical Lemmas}

Let $p_{s s^{\prime}}^{m}(\tau)$ denote the probability that type $m$ memory will be in state $s^{\prime}$ in a time interval of length $\tau>0$, given that the process is currently in state $s$ :

$$
p_{s s^{\prime}}^{m}(\tau) \equiv \operatorname{Pr}\left\{M_{t+\tau}=s^{\prime} \mid M_{t}=s\right\}
$$

where the dependence on $\alpha_{A}$ and $\alpha_{B}$ is suppressed for the moment. This probability is independent of $t$, since the memory process has the Markov property.

Lemma 2. (i) Suppose the memory process is currently in state $s_{\varnothing}$. Then,

$$
p_{s_{\varnothing} s_{i}}^{m}\left(\tau ; \alpha_{A}, \alpha_{B}\right)=\frac{\alpha_{i}\left(1-e^{-\left(\alpha_{A}+\alpha_{B}\right) \tau}\right)}{\alpha_{A}+\alpha_{B}},
$$

where $m=P, R$, and $i=A, B$. 
(ii) Suppose the memory process is currently in state $s_{i}$. Then,

$$
\begin{aligned}
& p_{s_{i} s_{i}}^{m}\left(\tau ; \alpha_{A}, \alpha_{B}\right)= \begin{cases}1, & \text { if } m=P, \\
\frac{\alpha_{i}+\alpha_{j} e^{-\left(\alpha_{A}+\alpha_{B}\right) \tau}}{\alpha_{A}+\alpha_{B}}, & \text { if } m=R,\end{cases} \\
& p_{s_{i} s_{j}}^{m}\left(\tau ; \alpha_{A}, \alpha_{B}\right)= \begin{cases}0, & \text { if } m=P, \\
\frac{\alpha_{j}\left(1-e^{-\left(\alpha_{A}+\alpha_{B}\right) \tau}\right)}{\alpha_{A}+\alpha_{B}}, & \text { if } m=R,\end{cases}
\end{aligned}
$$

where $i, j=A, B$, and $i \neq j$.

This technical lemma deserves some discussion. First, suppose the memory process is currently in state $s_{\varnothing}$. Probabilities $p_{s_{\varnothing} s_{A}}^{m}(\tau)$ and $p_{s_{\varnothing} s_{B}}^{m}(\tau)$ are obtained by applying $\phi=0$ to the formulas of Lemma 1 .

Next, suppose the process is in state $s_{i}, i=A, B$. Consider proactive memory: the only way the process can leave this state is through forgetting, which never happens as $\phi=0$. Therefore, for any $\tau>0, p_{s_{i} s_{i}}^{m}(\tau)=1$ and $p_{s_{i} s_{j}}^{m}(\tau)=0$.

Consider retroactive memory. It is easy to see that $p_{s_{i} s_{i}}^{m}(\tau)$ is always greater than the corresponding long-run probability $\bar{p}_{s_{i}}$, but converges to it as $\tau \rightarrow \infty$. In contrast, $p_{s_{i} s_{j}}^{m}(\tau)$ converges to $\bar{p}_{s_{j}}$ from below.

Define

$$
\left.V_{i}^{m}\left(\alpha_{A}, \alpha_{B}\right)\right|_{s}=\int_{0}^{\infty} e^{-r \tau} p_{s s_{i}}^{m}\left(\tau ; \alpha_{A}, \alpha_{B}\right) \sigma d \tau
$$

In other words, $\left.V_{i}^{m}\left(\alpha_{A}, \alpha_{B}\right)\right|_{s}$ is the gross (i.e., excluding the advertising costs) discounted profit function of firm $i$ given that the memory process is currently in state $s$.

Lemma 3. (i) Suppose that the memory process is currently in state $s_{\varnothing}$. Then,

$$
\left.V_{i}^{m}\left(\alpha_{A}, \alpha_{B}\right)\right|_{s \varnothing}=\frac{\sigma \alpha_{i}}{r\left(\alpha_{A}+\alpha_{B}+r\right)}
$$

where $m=P, R$, and $i=A, B$. 
(ii) Suppose that the memory process is currently in state $s_{i}$. Then,

$$
\begin{aligned}
\left.V_{i}^{m}\left(\alpha_{A}, \alpha_{B}\right)\right|_{s_{i}} & = \begin{cases}\frac{\sigma}{r}, & \text { if } m=P, \\
\frac{\sigma\left(\alpha_{i}+r\right)}{r\left(\alpha_{A}+\alpha_{B}+r\right)}, & \text { if } m=R,\end{cases} \\
\left.V_{j}^{m}\left(\alpha_{A}, \alpha_{B}\right)\right|_{s_{i}} & = \begin{cases}0, & \text { if } m=P, \\
\frac{\sigma \alpha_{j}}{r\left(\alpha_{A}+\alpha_{B}+r\right)}, & \text { if } m=R,\end{cases}
\end{aligned}
$$

where $i, j=A, B$, and $i \neq j$.

Consider proactive memory. If the memory process is currently in state $s_{i}$, firm $i$ 's gross profit is equal to $\sigma / r$ (maximum possible). The gross profit of its competitor is zero in this case.

When consumer memory is retroactive, the comparative advantage that firm $i$ has is less pronounced:

$$
\left.V_{i}^{R}\left(\alpha_{A}, \alpha_{B}\right)\right|_{s_{i}}=\frac{\sigma\left(\alpha_{i}+r\right)}{r\left(\alpha_{A}+\alpha_{B}+r\right)}<\frac{\sigma}{r}
$$

\section{$5 \quad$ Sequential Move Setting}

In this section the setting in which the firms make their advertising decisions sequentially is analyzed. Firm $A$ chooses its advertising frequency at the beginning of the game. Firm $B$ enters the market at some exogenous time $T>0$, observes firm $A$ 's advertising decision, and then chooses its own advertising frequency.

When firm $B$ enters the market, consumers of total mass $p_{s_{A}}\left(T ; \alpha_{A}, 0\right)=1-e^{-\alpha_{A} T}$ know about brand $A$, the rest (fraction $p_{s_{\varnothing}}\left(T ; \alpha_{A}, 0\right)=e^{-\alpha_{A} T}$ ) are not aware of the product existence. Lemmas 1 and 3 allow computation of the firms' profit functions. For $m=P, R$,

$$
\begin{aligned}
\Pi_{A}^{m}\left(\alpha_{A}, \alpha_{B}\right) & =\left.V_{A}^{m}\left(\alpha_{A}, 0\right)\right|_{s \varnothing}-\left.e^{-r T} \sum_{s=s_{\varnothing}, s_{A}} p_{s}\left(T ; \alpha_{A}, 0\right) V_{A}^{m}\left(\alpha_{A}, 0\right)\right|_{s} \\
& +\left.e^{-r T} \sum_{s=s_{\varnothing}, s_{A}} p_{s}\left(T ; \alpha_{A}, 0\right) V_{A}^{m}\left(\alpha_{A}, \alpha_{B}\right)\right|_{s}-\frac{c\left(\alpha_{A}\right)}{r}, \\
\Pi_{B}^{m}\left(\alpha_{A}, \alpha_{B}\right) & =\left.\sum_{s=s_{\varnothing}, s_{A}} p_{s}\left(T ; \alpha_{A}, 0\right) V_{B}^{m}\left(\alpha_{A}, \alpha_{B}\right)\right|_{s}-\frac{c\left(\alpha_{B}\right)}{r} .
\end{aligned}
$$


The first two terms of $\Pi_{A}^{m}$ constitute the gross discounted profit of firm $A$ before time $T$. It is equal to

$$
\frac{\sigma \alpha_{A}}{r\left(\alpha_{A}+r\right)}-e^{-r T}\left(e^{-\alpha_{A} T} \frac{\sigma \alpha_{A}}{r\left(\alpha_{A}+r\right)}+\left(1-e^{-\alpha_{A} T}\right)\right)
$$

and is the same for both types of consumer memory.

The third term of $\Pi_{A}^{m}$ is the gross profit of firm $A$ from time $T$ on, discounted to $t=0$. It is equal to

$$
e^{-r T}\left(e^{-\alpha_{A} T} \frac{\sigma \alpha_{A}}{r\left(\alpha_{A}+\alpha_{B}+r\right)}+\left(1-e^{-\alpha_{A} T}\right)\right)
$$

when memory is proactive, and to

$$
e^{-r T}\left(e^{-\alpha_{A} T} \frac{\sigma \alpha_{A}}{r\left(\alpha_{A}+\alpha_{B}+r\right)}+\left(1-e^{-\alpha_{A} T}\right) \frac{\sigma\left(\alpha_{A}+r\right)}{r\left(\alpha_{A}+\alpha_{B}+r\right)}\right)
$$

when memory is retroactive.

The first term of $\Pi_{B}^{m}$ is the gross discounted profit of firm $B$ from time $T$ on, discounted to $t=T$. It is equal to

$$
e^{-\alpha_{A} T} \frac{\sigma \alpha_{B}}{r\left(\alpha_{A}+\alpha_{B}+r\right)}
$$

when memory is proactive, and to

$$
\frac{\sigma \alpha_{B}}{r\left(\alpha_{A}+\alpha_{B}+r\right)}
$$

when memory is retroactive.

This serves as a proof of the following proposition.

Proposition 3 (Profit Functions). In the Sequential Move setting, the profit functions of the firms do depend on the type of consumer memory.

(i) When consumer memory is proactive,

$$
\begin{aligned}
& \Pi_{A}^{P}\left(\alpha_{A}, \alpha_{B}\right)=\frac{\sigma \alpha_{A}}{r\left(\alpha_{A}+r\right)}-\frac{c\left(\alpha_{A}\right)}{r}-e^{-r T} e^{-\alpha_{A} T} \frac{\sigma \alpha_{A} \alpha_{B}}{r\left(\alpha_{A}+r\right)\left(\alpha_{A}+\alpha_{B}+r\right)}, \\
& \Pi_{B}^{P}\left(\alpha_{A}, \alpha_{B}\right)=e^{-\alpha_{A} T} \frac{\sigma \alpha_{B}}{r\left(\alpha_{A}+\alpha_{B}+r\right)}-\frac{c\left(\alpha_{B}\right)}{r} .
\end{aligned}
$$


(ii) When consumer memory is retroactive,

$$
\begin{aligned}
\Pi_{A}^{R}\left(\alpha_{A}, \alpha_{B}\right) & =\frac{\sigma \alpha_{A}}{r\left(\alpha_{A}+r\right)}-\frac{c\left(\alpha_{A}\right)}{r}-e^{-r T} \frac{\sigma \alpha_{B}}{r\left(\alpha_{A}+\alpha_{B}+r\right)} \\
& +e^{-r T} e^{-\alpha_{A} T} \frac{\sigma \alpha_{B}}{\left(\alpha_{A}+r\right)\left(\alpha_{A}+\alpha_{B}+r\right)}, \\
\Pi_{B}^{R}\left(\alpha_{A}, \alpha_{B}\right) & =\frac{\sigma \alpha_{B}}{r\left(\alpha_{A}+\alpha_{B}+r\right)}-\frac{c\left(\alpha_{B}\right)}{r} .
\end{aligned}
$$

It follows that for any given values of $\alpha_{A}$ and $\alpha_{B}$, firm $A$ 's profit is higher when memory is proactive than when it is retroactive, while firm $B$ 's profit is higher when memory is retroactive than when it is proactive. Specifically,

$$
\Pi_{A}^{P}\left(\alpha_{A}, \alpha_{B}\right)-\Pi_{A}^{R}\left(\alpha_{A}, \alpha_{B}\right)=e^{-r T}\left(1-e^{-\alpha_{A} T}\right) \frac{\alpha_{B}}{\alpha_{A}+\alpha_{B}+r},
$$

which is exactly

$$
e^{-r T}\left(\Pi_{B}^{R}\left(\alpha_{A}, \alpha_{B}\right)-\Pi_{B}^{P}\left(\alpha_{A}, \alpha_{B}\right)\right)
$$

This is not a coincidence. Observe that the fraction of consumers that are aware of the product availability trough the firms' ads is independent of whether consumer memory is proactive or retroactive. Therefore, the sum of the firms' profit functions,

$$
\Pi_{A}^{m}\left(\alpha_{A}, \alpha_{B}\right)+e^{-r T} \Pi_{B}^{m}\left(\alpha_{A}, \alpha_{B}\right)
$$

is the same for $m=P, R$.

Proposition 4 (Firm B's Strategy). In the Sequential Move setting:

(i) When consumer memory is proactive, firm B's strategy, $\alpha_{B}^{P}\left(\alpha_{A}\right)$, is implicitly defined by

$$
e^{-\alpha_{A} T} \frac{\sigma\left(\alpha_{A}+r\right)}{\left(\alpha_{A}+\alpha_{B}^{P}+r\right)^{2}}=c^{\prime}\left(\alpha_{B}^{P}\right)
$$

(ii) When consumer memory is retroactive, firm B's strategy, $\alpha_{B}^{R}\left(\alpha_{A}\right)$, is implicitly defined 
by

$$
\frac{\sigma\left(\alpha_{A}+r\right)}{\left(\alpha_{A}+\alpha_{B}^{R}+r\right)^{2}}=c^{\prime}\left(\alpha_{B}^{R}\right) .
$$

(iii) For each value of $\alpha_{A}$, firm $B$ chooses lower advertising frequency when memory is proactive: $\alpha_{B}^{P}\left(\alpha_{A}\right)<\alpha_{B}^{R}\left(\alpha_{A}\right)$.

Firm $B$ has less incentive to invest in advertising when memory is proactive, since at time $T$ the fraction of its potential customers (i.e., those not already locked up by firm $A$ ) is only $e^{-\alpha_{A} T}$. When memory is retroactive, by contrast, firm $A$ cannot foreclose any of the market to firm $B$ because consumers will purchase according to the last ad they observe.

Proposition 5 (Firm A's Equilibrium Advertising Frequency). In the Sequential Move setting, if $T$ is large enough, firm A chooses higher advertising frequency when consumer memory is retroactive: $\alpha_{A}^{R}>\alpha_{A}^{P}$.

Sketch of the Proof. First, let $T=\infty$. Firm $A$ maximizes monopoly payoff

$$
\bar{\Pi}_{A}\left(\alpha_{A}\right) \equiv \frac{\sigma \alpha_{A}}{r\left(\alpha_{A}+r\right)}-\frac{c\left(\alpha_{A}\right)}{r}
$$

which is independent of whether consumer memory is proactive or retroactive. Let $\bar{\alpha}_{A}$ denote $\arg \max _{\alpha_{A}} \bar{\Pi}_{A}\left(\alpha_{A}\right)$.

Next, consider large but finite $T$. Firm $A$ maximizes

$$
\Pi_{A}^{P}\left(\alpha_{A}, \alpha_{B}^{P}\left(\alpha_{A}\right)\right)=\bar{\Pi}_{A}\left(\alpha_{A}\right)+\mathrm{O}\left(\mathrm{e}^{-\left(\mathrm{r}+\alpha_{\mathrm{A}}\right) \mathrm{T}}\right)
$$

when memory is proactive, and

$$
\Pi_{A}^{R}\left(\alpha_{A}, \alpha_{B}^{R}\left(\alpha_{A}\right)\right)=\bar{\Pi}_{A}\left(\alpha_{A}\right)+e^{-r T} \frac{\sigma \alpha_{B}^{R}\left(\alpha_{A}\right)}{r\left(\alpha_{A}+\alpha_{B}^{R}\left(\alpha_{A}\right)+r\right)}+\mathrm{O}\left(\mathrm{e}^{-\left(\mathrm{r}+\alpha_{\mathrm{A}}\right) \mathrm{T}}\right)
$$

when memory is retroactive. Here $\mathrm{O}\left(\mathrm{e}^{-\left(\mathrm{r}+\alpha_{\mathrm{A}}\right) \mathrm{T}}\right)$ is of the same order as $e^{-\left(r+\alpha_{A}\right) T}$. That is,

$$
0<\lim _{T \rightarrow \infty} \frac{\left|\mathrm{O}\left(\mathrm{e}^{-\left(\mathrm{r}+\alpha_{\mathrm{A}}\right) \mathrm{T}}\right)\right|}{e^{-\left(r+\alpha_{A}\right) T}}<\infty
$$


The marginal effect of increasing advertising frequency from monopoly level $\bar{\alpha}_{A}$ is negligibly small (of order $e^{-\left(r+\bar{\alpha}_{A}\right) T}$ ) when memory is proactive. The effect is sizable when memory is retroactive. Straightforward algebra (see the Appendix) shows that

$$
\left.\frac{d \Pi_{A}^{R}\left(\alpha_{A}, \alpha_{B}^{R}\left(\alpha_{A}\right)\right)}{d \alpha_{A}}\right|_{\bar{\alpha}_{A}}=e^{-r T} H\left(\bar{\alpha}_{A}\right)+\mathrm{O}\left(\mathrm{e}^{-\left(\mathrm{r}+\bar{\alpha}_{\mathrm{A}}\right) \mathrm{T}}\right)
$$

where $H\left(\bar{\alpha}_{A}\right)>0$. It follows that in equilibrium firm $A$ chooses higher advertising frequency when memory is retroactive.

\section{Dynamic Setting}

In this section, the setting in which the firms choose their advertising frequencies twice, at $t=0$ and at $t=T$, is analyzed.

Let $\alpha_{A 0}$ and $\alpha_{B 0}$ denote the firms' advertising frequencies chosen at time $0 ; \alpha_{A T}$ and $\alpha_{B T}$ denote the frequencies chosen at time $T$. Lemmas 1 and 3 allow computation of the firms' profit functions.

$$
\begin{aligned}
\Pi_{i}^{m} & =\left.V_{i}^{m}\left(\alpha_{A 0}, \alpha_{B 0}\right)\right|_{s \varnothing}-\left.e^{-r T} \sum_{s=s \varnothing, s_{A}, s_{B}} p_{s}\left(T ; \alpha_{A 0}, \alpha_{B 0}\right) V_{i}^{m}\left(\alpha_{A 0}, \alpha_{B 0}\right)\right|_{s} \\
& -\left(1-e^{-r T}\right) \frac{c\left(\alpha_{A 0}\right)}{r}+e^{-r T} \Pi_{i T}^{m}\left(\alpha_{A 0}, \alpha_{B 0}, \alpha_{A T}, \alpha_{B T}\right),
\end{aligned}
$$

where $m=P, R$, and $i=A, B$.

The first two terms constitute the gross discounted profit of firm $i$ from $t=0$ to $T$. It is equal to

$$
\frac{\sigma \alpha_{i 0}}{r\left(\alpha_{A 0}+\alpha_{B 0}+r\right)}-e^{-r T}\left(\frac{\sigma \alpha_{i 0} e^{-\left(\alpha_{A 0}+\alpha_{B 0}\right) T}}{r\left(\alpha_{A 0}+\alpha_{B 0}+r\right)}+\frac{\sigma \alpha_{i 0}\left(1-e^{-\left(\alpha_{A 0}+\alpha_{B 0}\right) T}\right)}{r\left(\alpha_{A 0}+\alpha_{B 0}\right)}\right)
$$

and is the same for both types of consumer memory. The last term, $e^{-r T} \Pi_{i T}^{m}$, is firm $i$ 's profit 
from time $T$ on, discounted to $t=0$.

$$
\begin{aligned}
\Pi_{i T}^{P} & =\left.\sum_{s=s_{\varnothing}, s_{A}, s_{B}} p_{s}\left(T ; \alpha_{A 0}, \alpha_{B 0}\right) V_{i}^{P}\left(\alpha_{A T}, \alpha_{B T}\right)\right|_{s}-\frac{c\left(\alpha_{A T}\right)}{r} \\
& =\frac{\sigma \alpha_{i T} e^{-\left(\alpha_{A 0}+\alpha_{B 0}\right) T}}{r\left(\alpha_{A T}+\alpha_{B T}+r\right)}+\frac{\sigma \alpha_{i 0}\left(1-e^{-\left(\alpha_{A 0}+\alpha_{B 0}\right) T}\right)}{r\left(\alpha_{A 0}+\alpha_{B 0}\right)}-\frac{c\left(\alpha_{i T}\right)}{r} \\
\Pi_{i T}^{R} & =\frac{\sigma \alpha_{i T} e^{-\left(\alpha_{A 0}+\alpha_{B 0}\right) T}}{r\left(\alpha_{A T}+\alpha_{B T}+r\right)}+\frac{\sigma\left(\alpha_{i T}+r\right) \alpha_{i 0}\left(1-e^{-\left(\alpha_{A 0}+\alpha_{B 0}\right) T}\right)}{r\left(\alpha_{A T}+\alpha_{B T}+r\right)\left(\alpha_{A 0}+\alpha_{B 0}\right)} \\
& +\frac{\sigma \alpha_{i T} \alpha_{j 0}\left(1-e^{-\left(\alpha_{A 0}+\alpha_{B 0}\right) T}\right)}{r\left(\alpha_{A T}+\alpha_{B T}+r\right)\left(\alpha_{A 0}+\alpha_{B 0}\right)}-\frac{c\left(\alpha_{i T}\right)}{r}
\end{aligned}
$$

This serves as a proof of the following proposition.

Proposition 6 (Profit Functions). In the Dynamic setting, the profit functions of the firms do depend on the type of consumer memory.

(i) When consumer memory is proactive,

$$
\begin{aligned}
\Pi_{i}^{P} & =\frac{\sigma \alpha_{i 0}}{r\left(\alpha_{A 0}+\alpha_{B 0}+r\right)}-\left(1-e^{-r T}\right) \frac{c\left(\alpha_{i 0}\right)}{r}-e^{-r T} \frac{c\left(\alpha_{i T}\right)}{r} \\
& \left.-e^{-r T} e^{-\left(\alpha_{A 0}+\alpha_{B 0}\right) T}\left(\frac{\alpha_{i 0}}{\alpha_{A 0}+\alpha_{B 0}+r}-\frac{\alpha_{i T}}{\alpha_{A T}+\alpha_{B T}+r}\right)\right)
\end{aligned}
$$

where $i=A, B$.

(ii) When consumer memory is retroactive,

$$
\Pi_{i}^{R}=\Pi_{i}^{P}-e^{-r T}\left(1-e^{-\left(\alpha_{A 0}+\alpha_{B 0}\right) T}\right) \frac{\sigma\left(\alpha_{i 0} \alpha_{j T}-\alpha_{j 0} \alpha_{i T}\right)}{r\left(\alpha_{A T}+\alpha_{B T}+r\right)\left(\alpha_{A 0}+\alpha_{B 0}\right)}
$$

where $i, j=A, B$, and $i \neq j$.

It follows that firm $i$ 's profit is higher when memory is proactive if and only if

$$
\alpha_{i 0} \alpha_{j T}-\alpha_{j 0} \alpha_{i T}>0
$$

or

$$
\alpha_{i 0} / \alpha_{j 0}>\alpha_{i T} / \alpha_{j T}
$$


This is very intuitive. Fix $\alpha_{i T}$ and $\alpha_{j T}$ for a moment. If $\alpha_{i 0}$ is high relative to $\alpha_{j 0}$ and memory is proactive, then, from time $T$ on, firm $i$ gets most of its profit from selling the product to large fraction $p_{i}\left(T ; \alpha_{A 0}, \alpha_{B 0}\right)$ of consumers (having received an ad describing firm $i$ 's product, these consumers will never switch to brand $j$ ).

If $\alpha_{i 0}$ is low relative to $\alpha_{j 0}$, then firm $i$ gets higher profit when memory is retroactive, since even consumers that are aware of brand $j$ at time $T$ (fraction $p_{j}\left(T ; \alpha_{A 0}, \alpha_{B 0}\right.$ ), which is large in this case) are among its potential buyers.

Also, observe that the sum of the firms' profit functions,

$$
\Pi_{A}^{m}\left(\alpha_{A}, \alpha_{B}\right)+e^{-r T} \Pi_{B}^{m}\left(\alpha_{A}, \alpha_{B}\right)
$$

is the same for $m=P, R$ (at any point in time, the fraction of consumers that know about the product is independent of whether consumer memory is proactive or retroactive).

Proposition 7 (Equilibrium Strategies at $t=T$ ). In the Dynamic setting:

(i) When consumer memory is proactive, the firms' equilibrium strategies at time $T, \alpha_{A T}^{P}\left(\alpha_{A 0}, \alpha_{B 0}\right)$ and $\alpha_{B T}^{P}\left(\alpha_{A 0}, \alpha_{B 0}\right)$, are implicitly defined by the system of equations

$$
\left\{\begin{array}{c}
e^{-\left(\alpha_{A 0}+\alpha_{B 0}\right) T} \frac{\sigma\left(\alpha_{B T}^{P}+r\right)}{\left(\alpha_{A T}^{P}+\alpha_{B T}^{P}+r\right)^{2}}=c^{\prime}\left(\alpha_{A T}^{P}\right), \\
e^{-\left(\alpha_{A 0}+\alpha_{B 0}\right) T} \frac{\sigma\left(\alpha_{A T}^{P}+r\right)}{\left(\alpha_{A T}^{P}+\alpha_{B T}^{P}+r\right)^{2}}=c^{\prime}\left(\alpha_{B T}^{P}\right) .
\end{array}\right.
$$

(ii) When consumer memory is retroactive, the firms' equilibrium strategies at time $T, \alpha_{A T}^{R}\left(\alpha_{A 0}, \alpha_{B 0}\right)$ and $\alpha_{B T}^{R}\left(\alpha_{A 0}, \alpha_{B 0}\right)$, are implicitly defined by the system of equations

$$
\left\{\begin{array}{l}
\frac{\sigma\left(\left(\alpha_{A 0}+\alpha_{B 0}\right) \alpha_{B T}^{R}+e^{-\left(\alpha_{A 0}+\alpha_{B 0}\right) T} \alpha_{A 0} r+\alpha_{B 0} r\right)}{\left(\alpha_{A 0}+\alpha_{B 0}\right)\left(\alpha_{A T}^{R}+\alpha_{B T}^{R}+r\right)^{2}}=c^{\prime}\left(\alpha_{A T}^{R}\right), \\
\frac{\sigma\left(\left(\alpha_{A 0}+\alpha_{B 0}\right) \alpha_{A T}^{R}+e^{-\left(\alpha_{A 0}+\alpha_{B 0}\right) T} \alpha_{B 0} r+\alpha_{A 0} r\right)}{\left(\alpha_{A 0}+\alpha_{B 0}\right)\left(\alpha_{A T}^{R}+\alpha_{B T}^{R}+r\right)^{2}}=c^{\prime}\left(\alpha_{B T}^{R}\right) .
\end{array}\right.
$$

Proposition 8 (Equilibrium Strategies at $t=0$ ). In the Dynamic setting, if $T$ is large enough, the firms choose higher advertising frequencies when consumer memory is proactive: $\alpha_{A 0}^{P}=\alpha_{B 0}^{P}>\alpha_{A 0}^{R}=\alpha_{B 0}^{R}$. 
Note that in the limit as $T \rightarrow \infty$ the Dynamic setting converges to the Simultaneous Move setting, in which case advertising frequencies chosen by the firms at $t=0$ do not depend on the type of memory.

Sketch of the Proof. Consider large but finite $T$. Incorporating time $T$ strategies into the profit functions yields

$$
\Pi_{i}^{P}=\frac{\sigma \alpha_{i 0}}{r\left(\alpha_{A 0}+\alpha_{B 0}+r\right)}-\left(1-e^{-r T}\right) \frac{c\left(\alpha_{i 0}\right)}{r}-e^{-r T} \frac{c\left(\alpha_{i T}^{P}\right)}{r}+\mathrm{O}\left(\mathrm{e}^{-\left(\alpha_{\mathrm{A} 0}+\alpha_{\mathrm{B} 0}+\mathrm{r}\right) \mathrm{T}}\right)
$$

when memory is proactive, and

$$
\begin{aligned}
\Pi_{i}^{R} & =\frac{\sigma \alpha_{i 0}}{r\left(\alpha_{A 0}+\alpha_{B 0}+r\right)}-\left(1-e^{-r T}\right) \frac{c\left(\alpha_{i 0}\right)}{r}-e^{-r T} \frac{c\left(\alpha_{i T}^{R}\right)}{r} \\
& -e^{-r T} \frac{\sigma\left(\alpha_{i 0} \alpha_{j T}^{R}-\alpha_{j 0} \alpha_{i T}^{R}\right)}{r\left(\alpha_{A 0}+\alpha_{B 0}\right)\left(\alpha_{A T}^{R}+\alpha_{B T}^{R}+r\right)}+\mathrm{O}\left(\mathrm{e}^{-\left(\alpha_{\mathrm{A} 0}+\alpha_{\mathrm{B} 0}+\mathrm{r}\right) \mathrm{T}}\right)
\end{aligned}
$$

when memory is retroactive. In the symmetric equilibrium the firms choose advertising frequencies $\alpha_{A 0}^{m}=\alpha_{B 0}^{m}=\alpha_{0}^{m}$, where $\alpha_{0}^{m}$ satisfies

$$
\left.\left.\frac{d \Pi_{i}^{m}}{d \alpha_{i 0}}\right|_{\alpha_{i 0}=\alpha_{j 0}=\alpha_{0}^{m}} \equiv\left(\frac{\partial \Pi_{i}^{m}}{\partial \alpha_{i 0}}+\frac{\partial \Pi_{i}^{m}}{\partial \alpha_{i T}^{m}} \frac{d \alpha_{i T}^{m}}{d \alpha_{i 0}}+\frac{\partial \Pi_{i}^{m}}{\partial \alpha_{j T}^{m}} \frac{d \alpha_{j T}^{m}}{d \alpha_{i 0}}\right)\right|_{\alpha_{i 0}=\alpha_{j 0}=\alpha_{0}^{m}}=0
$$

The second term in brackets is zero, as $\alpha_{i T}^{m}$ is chosen optimally. Performing simple but, in the case of retroactive memory, tedious algebra yields the following equations for $\alpha_{0}^{P}$ and $\alpha_{0}^{R}$ :

$$
\frac{\sigma\left(\alpha_{0}^{P}+r\right)}{\left(2 \alpha_{0}^{P}+r\right)^{2}}-\left(1-e^{-r T}\right) c^{\prime}\left(\alpha_{0}^{P}\right)+\mathrm{O}\left(\mathrm{e}^{-\left(2 \alpha_{0}^{\mathrm{P}}+\mathrm{r}\right) \mathrm{T}}\right)=0
$$

and

$$
\frac{\sigma\left(\alpha_{0}^{R}+r\right)}{\left(2 \alpha_{0}^{R}+r\right)^{2}}-\left(1-e^{-r T}\right) c^{\prime}\left(\alpha_{0}^{R}\right)-e^{-r T} G\left(\alpha_{0}^{R}\right)+\mathrm{O}\left(\mathrm{e}^{-\left(2 \alpha_{0}^{\mathrm{R}}+\mathrm{r}\right) \mathrm{T}}\right)=0,
$$

where $G\left(\alpha_{0}^{R}\right)>0$ (see the Appendix). It follows that in equilibrium the firms choose higher advertising frequencies when memory is proactive: $\alpha_{0}^{P}>\alpha_{0}^{R}$. 


\section{Conclusion}

This paper has applied the theories of proactive and retroactive interference developed in the psychology literature to explore how consumer memory affects advertising competition between firms. The equilibrium advertising frequencies were characterized under three different strategic settings: simultaneous, sequential, and dynamic moves.

It was shown that when firms choose their advertising frequencies simultaneously, the equilibrium outcome remarkably does not depend on whether memory corresponds to proactive or retroactive interference. The equilibrium outcome in this setting exhibits excessive advertising due to a type of brand-stealing effect.

In the Sequential Move and Dynamic settings, proactive interference was shown to have similar competitive effects as consumer switching $\operatorname{costs}^{9}$. Indeed, under proactive interference a consumer remembers the brand that was first to capture her attention through an advertisement. Moreover, this exposure hampers her ability to learn subsequently about competing brands. In the Sequential Move setting, the second firm chooses lower advertising frequency when memory is proactive than when it is retroactive, since by the time it enters the market, many consumers have already been locked up by the first firm. In the Dynamic setting, the firms compete for consumers very aggressively at the beginning of the game when memory is proactive. By the time the firms reconsider their advertising strategies, the fraction of consumers that have not been locked up is small, and the firms consequently choose relatively low levels of advertising for the rest of the game.

It was assumed that consumers could never remember more than a single brand at a time which implied no scope for price competition. Specifically, each firm charges the monopoly price to the consumers who remember its ad. A natural extension of the model would be to introduce additional memory registers and firms. For instance, suppose there were three firms and consumers could remember two brands. Then, the firms would compete not only in advertising, but also in prices. Another important extension would be to introduce a role for brand familiarity. (Familiar brands are less sensitive to interference effects.) This could be

\footnotetext{
${ }^{9}$ On switching costs see, for example, Klemperer's (1987) pioneering article and his subsequent (1995) survey.
} 
modelled by supposing that consumers were more likely to remember brands that they had used in the past.

The study of the economic consequences of bounded memory is still in its infancy ${ }^{10}$. This paper has made a first attempt at understanding how the limited memory of consumers might impact the advertising strategies of firms. While several important themes emerged from this investigation, there are clearly many avenues for future research in which the insights gleaned here may be refined and extended.

\footnotetext{
${ }^{10}$ See, for example, Wilson (2002), Mullainathan (2002), and Krähmer (2004).
} 


\section{Appendix}

\section{Proof of Proposition 1.}

For $i=A, B$,

$$
\begin{aligned}
\Pi_{i}\left(\alpha_{A}, \alpha_{B}\right) & =\int_{0}^{\infty} e^{-r t} p_{s_{i}}\left(t ; \alpha_{A}, \alpha_{B}\right) \sigma d t-\frac{c\left(\alpha_{i}\right)}{r} \\
& =\frac{\sigma \alpha_{i}}{\alpha_{A}+\alpha_{B}+\phi} \int_{0}^{\infty}\left(e^{-r t}-e^{-\left(\alpha_{A}+\alpha_{B}+r+\phi\right) t}\right) d t-\frac{c\left(\alpha_{i}\right)}{r} \\
& =\frac{\sigma \alpha_{i}}{\alpha_{A}+\alpha_{B}+\phi}\left(\frac{1}{r}-\frac{1}{\alpha_{A}+\alpha_{B}+r+\phi}\right)-\frac{c\left(\alpha_{i}\right)}{r} \\
& =\frac{\sigma \alpha_{i}}{r\left(\alpha_{A}+\alpha_{B}+r+\phi\right)}-\frac{c\left(\alpha_{i}\right)}{r} .
\end{aligned}
$$

\section{Proof of Proposition 2.}

Each part is proven in turn.

(i) Social planner solves

$$
\max _{\alpha_{A}, \alpha_{B}} \Pi_{A}\left(\alpha_{A}, \alpha_{B}\right)+\Pi_{B}\left(\alpha_{A}, \alpha_{B}\right),
$$

or

$$
\begin{gathered}
\max _{\alpha_{A}, \alpha_{B}} \frac{\sigma\left(\alpha_{A}+\alpha_{B}\right)}{r\left(\alpha_{A}+\alpha_{B}+r+\phi\right)}-\frac{c\left(\alpha_{A}\right)}{r}-\frac{c\left(\alpha_{B}\right)}{r}, \\
\text { F.O.C.: }\left\{\begin{array}{l}
\frac{\sigma(r+\phi)}{\left(\alpha_{A}+\alpha_{B}+r+\phi\right)^{2}}=c^{\prime}\left(\alpha_{A}\right), \\
\frac{\sigma(r+\phi)}{\left(\alpha_{A}+\alpha_{B}+r+\phi\right)^{2}}=c^{\prime}\left(\alpha_{B}\right) .
\end{array}\right.
\end{gathered}
$$

It is easy to show that the matrix of second derivatives

$$
\left(\begin{array}{cc}
-\frac{2 \sigma(r+\phi)}{r\left(\alpha_{A}+\alpha_{B}+r+\phi\right)^{3}}-\frac{c^{\prime \prime}\left(\alpha_{A}\right)}{r} & -\frac{2 \sigma(r+\phi)}{r\left(\alpha_{A}+\alpha_{B}+r+\phi\right)^{3}} \\
-\frac{2 \sigma(r+\phi)}{r\left(\alpha_{A}+\alpha_{B}+r+\phi\right)^{3}} & -\frac{2 \sigma(r+\phi)}{r\left(\alpha_{A}+\alpha_{B}+r+\phi\right)^{3}}-\frac{c^{\prime \prime}\left(\alpha_{B}\right)}{r}
\end{array}\right)
$$

is negative semidefinite, which implies sufficiency of the first-order conditions. Therefore, $\alpha_{A}^{\dagger}=\alpha_{B}^{\dagger}=\alpha^{\dagger}$, where $\alpha^{\dagger}$ satisfies

$$
\frac{\sigma(r+\phi)}{\left(2 \alpha^{\dagger}+r+\phi\right)^{2}}=c^{\prime}\left(\alpha^{\dagger}\right) .
$$


(ii) Firm $A$ 's best-response function $B R_{A}\left(\alpha_{B}\right)$ is a solution to

$$
\max _{\alpha_{A}} \Pi_{A}\left(\alpha_{A}, \alpha_{B}\right)
$$

or

$$
\begin{gathered}
\max _{\alpha_{A}} \frac{\sigma \alpha_{A}}{r\left(\alpha_{A}+\alpha_{B}+r+\phi\right)}-\frac{c\left(\alpha_{A}\right)}{r}, \\
\text { F.O.C.: } \frac{\sigma\left(\alpha_{B}+r+\phi\right)}{\left(\alpha_{A}+\alpha_{B}+r+\phi\right)^{2}}=c^{\prime}\left(\alpha_{A}\right) .
\end{gathered}
$$

As

$$
\frac{\partial^{2}}{\partial \alpha_{A}^{2}} \Pi\left(\alpha_{A}, \alpha_{B}\right)=\frac{-2 \sigma\left(\alpha_{B}+r+\phi\right)}{r\left(\alpha_{A}+\alpha_{B}+r+\phi\right)^{3}}-\frac{c^{\prime \prime}\left(\alpha_{A}\right)}{r}<0,
$$

the first-order conditions are sufficient. Similarly, $B R_{B}\left(\alpha_{A}\right)$ can be found from

$$
\frac{\sigma\left(\alpha_{A}+r+\phi\right)}{\left(\alpha_{A}+\alpha_{B}+r+\phi\right)^{2}}=c^{\prime}\left(\alpha_{B}\right)
$$

A solution to

$$
\left\{\begin{array}{c}
\frac{\sigma\left(\alpha_{B}+r+\phi\right)}{\left(\alpha_{A}+\alpha_{B}+r+\phi\right)^{2}}=c^{\prime}\left(\alpha_{A}\right), \\
\frac{\sigma\left(\alpha_{A}+r+\phi\right)}{\left(\alpha_{A}+\alpha_{B}+r+\phi\right)^{2}}=c^{\prime}\left(\alpha_{B}\right) .
\end{array}\right.
$$

yields equilibrium advertising frequencies. Uniqueness and symmetry of the solution is shown in two steps.

From the system above, it follows

$$
\left(\alpha_{A}+r+\phi\right) c^{\prime}\left(\alpha_{A}\right)=\left(\alpha_{B}+r+\phi\right) c^{\prime}\left(\alpha_{B}\right)
$$

The LHS (RHS) is increasing in $\alpha_{A}\left(\alpha_{B}\right)$. This observation implies the solution is symmetric, $\alpha_{A}^{*}=\alpha_{B}^{*}=\alpha^{*}$, where $\alpha^{*}$ satisfies

$$
\frac{\sigma\left(\alpha^{*}+r+\phi\right)}{\left(2 \alpha^{*}+r+\phi\right)^{2}}=c^{\prime}\left(\alpha^{*}\right) .
$$


Second,

$$
\begin{aligned}
B R_{A}^{\prime}\left(\alpha_{B}\right) & =\frac{2 \sigma\left(\alpha_{A}-\alpha_{B}-r-\phi\right)}{2 \sigma\left(\alpha_{B}+r+\phi\right)+\left(\alpha_{A}+\alpha_{B}+r+\phi\right)^{3} c^{\prime \prime}\left(\alpha_{A}\right)}, \\
B R_{B}^{\prime}\left(\alpha_{A}\right) & =\frac{2 \sigma\left(\alpha_{B}-\alpha_{A}-r-\phi\right)}{2 \sigma\left(\alpha_{A}+r+\phi\right)+\left(\alpha_{A}+\alpha_{B}+r+\phi\right)^{3} c^{\prime \prime}\left(\alpha_{B}\right)} .
\end{aligned}
$$

Observe that $\left.B R_{A}^{\prime}\left(\alpha_{B}\right)\right|_{\alpha_{A}=\alpha_{B}}$ and $\left.B R_{B}^{\prime}\left(\alpha_{A}\right)\right|_{\alpha_{A}=\alpha_{B}}<0$. Therefore, the solution is unique.

(iii) The LHS's of the equations that define $\alpha^{\dagger}$ and $\alpha^{*}$,

$$
\left(2 \alpha^{\dagger}+r+\phi\right)^{2} c^{\prime}\left(\alpha^{\dagger}\right)=\sigma(r+\phi)
$$

and

$$
\left(2 \alpha^{*}+r+\phi\right)^{2} c^{\prime}\left(\alpha^{*}\right)=\sigma\left(\alpha^{*}+r+\phi\right) \text {, }
$$

are increasing functions of $\alpha^{\dagger}$ and $\alpha^{*}$, respectively. This observation implies $\alpha^{*}>\alpha^{\dagger}$.

\section{Proof of Lemma 2.}

Each part is proven in turn.

(i) See Lemma 1.

(ii) When memory is proactive, $p_{s_{i} s_{i}}^{P}(\tau)=1, p_{s_{i} s_{j}}^{P}(\tau)=0$. When memory is retroactive, probabilities $p_{s_{i} s_{A}}^{R}(\tau)$ and $p_{s_{i} s_{A}}^{R}(\tau)$ satisfy the following system of linear differential equations:

$$
\left\{\begin{array}{l}
\frac{d}{d \tau} p_{s_{i} s_{A}}^{R}=-\alpha_{B} p_{s_{i} s_{A}}^{R}+\alpha_{A} p_{s_{i} s_{B}}^{R} \\
\frac{d}{d \tau} p_{s_{i} s_{B}}^{R}=-\alpha_{A} p_{s_{i} s_{B}}^{R}+\alpha_{B} p_{s_{i} s_{A}}^{R}
\end{array}\right.
$$

with initial conditions $p_{s_{i} s_{i}}^{R}(0)=1, p_{s_{i} s_{j}}^{R}(0)=0$.

The derivation will appear in the next draft of this paper. 


\section{Proof of Lemma 3.}

Each part is proven in turn.

(i) For $i=A, B$,

$$
\begin{aligned}
\left.V_{i}^{m}\left(\alpha_{A}, \alpha_{B}\right)\right|_{s \varnothing} & =\int_{0}^{\infty} e^{-r \tau} p_{s \varnothing}^{m}\left(\alpha_{A}, \alpha_{B}\right) \sigma d \tau \\
& =\frac{\sigma \alpha_{i}}{\alpha_{A}+\alpha_{B}} \int_{0}^{\infty}\left(e^{-r \tau}-e^{-\left(\alpha_{A}+\alpha_{B}+r\right) \tau}\right) d \tau \\
& =\frac{\sigma \alpha_{i}}{\alpha_{A}+\alpha_{B}}\left(\frac{1}{r}-\frac{1}{\alpha_{A}+\alpha_{B}+r}\right)=\frac{\sigma \alpha_{i}}{r\left(\alpha_{A}+\alpha_{B}+r\right)}
\end{aligned}
$$

(ii) When memory is proactive, $\left.V_{i}^{P}\left(\alpha_{A}, \alpha_{B}\right)\right|_{s_{i}}=\sigma / r$ and $\left.V_{j}^{P}\left(\alpha_{A}, \alpha_{B}\right)\right|_{s_{i}}=0$. When memory is retroactive,

$$
\begin{aligned}
\left.V_{i}^{R}\left(\alpha_{A}, \alpha_{B}\right)\right|_{s_{i}} & =\int_{0}^{\infty} e^{-r \tau} p_{s_{i} s_{i}}^{R}\left(\alpha_{A}, \alpha_{B}\right) \sigma d \tau \\
& =\frac{\sigma}{\alpha_{A}+\alpha_{B}} \int_{0}^{\infty}\left(\alpha_{i} e^{-r \tau}+\alpha_{j} e^{-\left(\alpha_{A}+\alpha_{B}+r\right) \tau}\right) d \tau \\
& =\frac{\sigma}{\alpha_{A}+\alpha_{B}}\left(\frac{\alpha_{i}}{r}+\frac{\alpha_{j}}{\alpha_{A}+\alpha_{B}+r}\right)=\frac{\sigma\left(\alpha_{i}+r\right)}{r\left(\alpha_{A}+\alpha_{B}+r\right)} \\
\left.V_{j}^{m}\left(\alpha_{A}, \alpha_{B}\right)\right|_{s_{i}} & =\int_{0}^{\infty} e^{-r \tau} p_{s_{i} s_{j}}^{R}\left(\alpha_{A}, \alpha_{B}\right) \sigma d \tau \\
& =\frac{\sigma \alpha_{j}}{\alpha_{A}+\alpha_{B}} \int_{0}^{\infty}\left(e^{-r \tau}-e^{-\left(\alpha_{A}+\alpha_{B}+r\right) t}\right) d \tau \\
& =\frac{\sigma \alpha_{j}}{\alpha_{A}+\alpha_{B}}\left(\frac{1}{r}-\frac{1}{\alpha_{A}+\alpha_{B}+r}\right)=\frac{\sigma \alpha_{j}}{r\left(\alpha_{A}+\alpha_{B}+r\right)} .
\end{aligned}
$$

\section{Proof of Proposition 4.}

Each part is proven in turn.

(i) When memory is proactive, firm $B$ solves

$$
\max _{\alpha_{B}} \Pi_{B}^{P}\left(\alpha_{A}, \alpha_{B}\right)
$$

or

$$
\max _{\alpha_{B}} e^{-\alpha_{A} T} \frac{\sigma \alpha_{B}}{r\left(\alpha_{A}+\alpha_{B}+r\right)}-\frac{c\left(\alpha_{B}\right)}{r}
$$




$$
\text { F.O.C.: } \quad e^{-\alpha_{A} T} \frac{\sigma\left(\alpha_{A}+r\right)}{\left(\alpha_{A}+\alpha_{B}^{P}+r\right)^{2}}=c^{\prime}\left(\alpha_{B}^{P}\right)
$$

As

$$
\frac{\partial^{2}}{\partial \alpha_{B}^{2}} \Pi_{B}^{m}\left(\alpha_{A}, \alpha_{B}\right)=-e^{-\alpha_{A} T} \frac{2 \sigma\left(\alpha_{A}+r\right)}{r\left(\alpha_{A}+\alpha_{B}^{P}+r\right)^{3}}-\frac{c^{\prime \prime}\left(\alpha_{B}^{P}\right)}{r}<0,
$$

the first-order conditions for $\alpha_{B}^{P}\left(\alpha_{A}\right)$ are sufficient.

(ii) Similarly, $\alpha_{B}^{R}\left(\alpha_{A}\right)$ is a solution to

$$
\frac{\sigma\left(\alpha_{A}+r\right)}{\left(\alpha_{A}+\alpha_{B}^{R}+r\right)^{2}}=c^{\prime}\left(\alpha_{B}^{R}\right)
$$

(iii) Rewrite the first-order conditions for $\alpha_{B}^{P}$ and $\alpha_{B}^{R}$ as

$$
\left(\alpha_{A}+\alpha_{B}^{P}+r\right)^{2} c^{\prime}\left(\alpha_{B}^{P}\right)=e^{-\alpha_{A} T} \sigma\left(\alpha_{A}+r\right)
$$

and

$$
\left(\alpha_{A}+\alpha_{B}^{R}+r\right)^{2} c^{\prime}\left(\alpha_{B}^{R}\right)=\sigma\left(\alpha_{A}+r\right)
$$

The LHS's of the above equations are increasing functions of $\alpha_{B}^{P}$ and $\alpha_{B}^{R}$, respectively.

This observation implies $\alpha_{B}^{P}\left(\alpha_{A}\right)<\alpha_{B}^{R}\left(\alpha_{A}\right)$, for each value of $\alpha_{A}$.

\section{Proof of Proposition 5.}

Consider large but finite $T$. Evaluating $\frac{d}{d \alpha_{A}} \Pi_{A}^{m}\left(\alpha_{A}, \alpha_{B}^{m}\left(\alpha_{A}\right)\right)$ at $\bar{\alpha}_{A}$ yields

$$
\left.\frac{d \Pi_{A}^{P}}{d \alpha_{A}}\right|_{\bar{\alpha}_{A}}=\mathrm{O}\left(\mathrm{e}^{-\left(\mathrm{r}+\bar{\alpha}_{\mathrm{A}}\right) \mathrm{T}}\right)
$$

and

$$
\begin{aligned}
\left.\frac{d \Pi_{A}^{R}}{d \alpha_{A}}\right|_{\bar{\alpha}_{A}} & =e^{-r T}\left(\frac{\sigma \alpha_{B}^{R}\left(\bar{\alpha}_{A}\right)}{r\left(\bar{\alpha}_{A}+\alpha_{B}^{R}\left(\bar{\alpha}_{A}\right)+r\right)^{2}}\right. \\
& \left.-\frac{\sigma\left(\bar{\alpha}_{A}+r\right)}{r\left(\bar{\alpha}_{A}+\alpha_{B}^{R}\left(\bar{\alpha}_{A}\right)+r\right)^{2}} \frac{d \alpha_{B}^{R}\left(\bar{\alpha}_{A}\right)}{d \bar{\alpha}_{A}}\right)+\mathrm{O}\left(\mathrm{e}^{-\left(\mathrm{r}+\bar{\alpha}_{\mathrm{A}}\right) \mathrm{T}}\right) .
\end{aligned}
$$


Applying the Implicit function theorem to equation (ii) of Proposition 4 yields

$$
\frac{d \alpha_{B}^{R}\left(\bar{\alpha}_{A}\right)}{d \bar{\alpha}_{A}}=-\frac{\bar{\alpha}_{A}+r-\alpha_{B}^{R}\left(\bar{\alpha}_{A}\right)}{2\left(\bar{\alpha}_{A}+r\right)+h\left(\bar{\alpha}_{A}\right)}
$$

where

$$
h\left(\bar{\alpha}_{A}\right) \equiv \frac{1}{\sigma} c^{\prime \prime}\left(\alpha_{B}^{R}\left(\bar{\alpha}_{A}\right)\right)\left(\bar{\alpha}_{A}+\alpha_{B}^{R}\left(\bar{\alpha}_{A}\right)+r\right)^{3}>0 .
$$

Thus,

$$
\begin{aligned}
\left.\frac{d \Pi_{A}^{R}}{d \alpha_{A}}\right|_{\bar{\alpha}_{A}} & =e^{-r T}\left(\frac{\sigma \alpha_{B}^{R}\left(\bar{\alpha}_{A}\right)}{r\left(\bar{\alpha}_{A}+\alpha_{B}^{R}\left(\bar{\alpha}_{A}\right)+r\right)^{2}}\right. \\
& \left.+\frac{\sigma\left(\bar{\alpha}_{A}+r\right)\left(\bar{\alpha}_{A}+r-\alpha_{B}^{R}\left(\bar{\alpha}_{A}\right)\right)}{r\left(\bar{\alpha}_{A}+\alpha_{B}^{R}\left(\bar{\alpha}_{A}\right)+r\right)^{2}\left(2\left(\bar{\alpha}_{A}+r\right)+h\left(\bar{\alpha}_{A}\right)\right)}\right)+\mathrm{O}\left(\mathrm{e}^{-\left(\mathrm{r}+\bar{\alpha}_{\mathrm{A}}\right) \mathrm{T}}\right) \\
& =e^{-r T} \frac{\left(\bar{\alpha}_{A}+r\right)\left(\bar{\alpha}_{A}+\alpha_{B}^{R}\left(\bar{\alpha}_{A}\right)+r\right)+\alpha_{B}^{R}\left(\bar{\alpha}_{A}\right) h\left(\bar{\alpha}_{A}\right)}{r\left(\bar{\alpha}_{A}+\alpha_{B}^{R}\left(\bar{\alpha}_{A}\right)+r\right)^{2}\left(2\left(\bar{\alpha}_{A}+r\right)+h\left(\bar{\alpha}_{A}\right)\right)}+\mathrm{O}\left(\mathrm{e}^{-\left(\mathrm{r}+\bar{\alpha}_{\mathrm{A}}\right) \mathrm{T}}\right) .
\end{aligned}
$$

It follows from the above that

$$
\left.\frac{d \Pi_{A}^{R}}{d \alpha_{A}}\right|_{\bar{\alpha}_{A}}>\left.\frac{d \Pi_{A}^{P}}{d \alpha_{A}}\right|_{\bar{\alpha}_{A}} .
$$

Therefore, firm $A$ chooses higher advertising frequency when memory is retroactive.

\section{Proof of Proposition 7.}

Each part is proven in turn.

(i) Consider proactive memory, $t=T$. Firm $i$ 's best-response function is a solution to

$$
\begin{gathered}
\max _{\alpha_{i T}} \Pi_{i T}^{P}\left(\alpha_{A 0}, \alpha_{B 0}, \alpha_{B 0}, \alpha_{B T}\right), \\
\text { F.O.C.: } e^{-\left(\alpha_{A 0}+\alpha_{B 0}\right) T} \frac{\sigma\left(\alpha_{j T}+r\right)}{\left(\alpha_{A T}+\alpha_{B T}+r\right)^{2}}=c^{\prime}\left(\alpha_{A T}\right) .
\end{gathered}
$$

As

$$
\frac{\partial^{2}}{\partial \alpha_{i T}^{2}} \Pi_{i T}^{P}=-e^{-\left(\alpha_{A 0}+\alpha_{B 0}\right) T} \frac{2 \sigma\left(\alpha_{j T}+r\right)}{r\left(\alpha_{A T}+\alpha_{B T}+r\right)^{3}}-\frac{c^{\prime \prime}\left(\alpha_{A T}\right)}{r}<0
$$


the first-order conditions are sufficient. A solution to

$$
\left\{\begin{array}{c}
e^{-\left(\alpha_{A 0}+\alpha_{B 0}\right) T} \frac{\sigma\left(\alpha_{B T}^{P}+r\right)}{\left(\alpha_{A T}^{P}+\alpha_{B T}^{P}+r\right)^{2}}=c^{\prime}\left(\alpha_{A T}^{P}\right), \\
e^{-\left(\alpha_{A 0}+\alpha_{B 0}\right) T} \frac{\sigma\left(\alpha_{A T}^{P}+r\right)}{\left(\alpha_{A T}^{P}+\alpha_{B T}^{P}+r\right)^{2}}=c^{\prime}\left(\alpha_{B T}^{P}\right) .
\end{array}\right.
$$

yields time $T$ equilibrium strategies, $\alpha_{A T}^{P}\left(\alpha_{A 0}, \alpha_{B 0}\right)$ and $\alpha_{B T}^{P}\left(\alpha_{A 0}, \alpha_{B 0}\right)$.

(ii) Consider retroactive memory, $t=T$. Firm $i$ 's best-response function is a solution to

$$
\max _{\alpha_{i T}} \Pi_{i T}^{R}\left(\alpha_{A 0}, \alpha_{B 0}, \alpha_{B 0}, \alpha_{B T}\right)
$$

F.O.C.:

$$
\begin{gathered}
\frac{\sigma\left(\alpha_{j T}+r\right) e^{-\left(\alpha_{A 0}+\alpha_{B 0}\right) T}}{\left(\alpha_{A T}+\alpha_{B T}+r\right)^{2}}+\frac{\sigma \alpha_{j T} \alpha_{i 0}\left(1-e^{-\left(\alpha_{A 0}+\alpha_{B 0}\right) T}\right)}{\left(\alpha_{A T}+\alpha_{B T}+r\right)^{2}\left(\alpha_{A 0}+\alpha_{B 0}\right)} \\
+\frac{\sigma\left(\alpha_{j T}+r\right) \alpha_{j 0}\left(1-e^{-\left(\alpha_{A 0}+\alpha_{B 0}\right) T}\right)}{\left(\alpha_{A T}+\alpha_{B T}+r\right)^{2}\left(\alpha_{A 0}+\alpha_{B 0}\right)}=c^{\prime}\left(\alpha_{A T}\right) .
\end{gathered}
$$

As $\partial^{2} \Pi_{i T}^{R} / \partial \alpha_{A T}^{2}<0$, the first-order conditions are sufficient. A solution to

$$
\left\{\begin{array}{l}
\frac{\sigma\left(\left(\alpha_{A 0}+\alpha_{B 0}\right) \alpha_{B T}^{R}+e^{-\left(\alpha_{A 0}+\alpha_{B 0}\right) T} \alpha_{A 0} r+\alpha_{B 0} r\right)}{\left(\alpha_{A 0}+\alpha_{B 0}\right)\left(\alpha_{A T}^{R}+\alpha_{B T}^{R}+r\right)^{2}}=c^{\prime}\left(\alpha_{A T}^{R}\right) \\
\frac{\sigma\left(\left(\alpha_{A 0}+\alpha_{B 0}\right) \alpha_{A T}^{R}+e^{-\left(\alpha_{A 0}+\alpha_{B 0}\right) T} \alpha_{B 0} r+\alpha_{A 0} r\right)}{\left(\alpha_{A 0}+\alpha_{B 0}\right)\left(\alpha_{A T}^{R}+\alpha_{B T}^{R}+r\right)^{2}}=c^{\prime}\left(\alpha_{B T}^{R}\right) .
\end{array}\right.
$$

yields time $T$ equilibrium strategies, $\alpha_{A T}^{R}\left(\alpha_{A 0}, \alpha_{B 0}\right)$ and $\alpha_{B T}^{R}\left(\alpha_{A 0}, \alpha_{B 0}\right)$.

\section{Proof of Proposition 8.}

Differentiating the system of equations that jointly defines $\alpha_{A T}^{R}$ and $\alpha_{B T}^{R}$ (part (ii) of Proposition 7) with respect to $\alpha_{i 0}$ yields

$$
\left.\frac{d \alpha_{j T}^{R}}{d \alpha_{i 0}}\right|_{\alpha_{i 0}=\alpha_{j 0}=\alpha_{0}}=\frac{r c^{\prime}\left(\alpha_{T}^{R}\right)}{2\left(2 \alpha_{0} c^{\prime}\left(\alpha_{T}^{R}\right)+\left(2 \alpha_{0} \alpha_{T}^{R}+\alpha_{0} r\right) c^{\prime \prime}\left(\alpha_{T}^{R}\right)\right)}+\mathrm{O}\left(\mathrm{e}^{-2 \alpha_{0} \mathrm{~T}}\right)
$$

where $\alpha_{T}^{R} \equiv \alpha_{A T}^{R}\left(\alpha_{0}, \alpha_{0}\right)=\alpha_{B T}^{R}\left(\alpha_{0}, \alpha_{0}\right)$. 
Next,

$$
\begin{aligned}
\left.\frac{d \Pi_{i}^{R}}{d \alpha_{i 0}}\right|_{\alpha_{i 0}=\alpha_{j 0}=\alpha_{0}} & =\frac{\sigma\left(\alpha_{0}+r\right)}{r\left(2 \alpha_{0}+r\right)^{2}}-\left(1-e^{-r T}\right) \frac{c^{\prime}\left(\alpha_{0}\right)}{r} \\
& -e^{-r T}\left(\frac{\sigma \alpha_{T}^{R}}{2 r \alpha_{0}\left(2 \alpha_{T}^{R}+r\right)}+\left.\frac{\sigma}{2 r\left(2 \alpha_{T}^{R}+r\right)} \frac{d \alpha_{j T}^{R}}{d \alpha_{i 0}}\right|_{\alpha_{i 0}=\alpha_{j 0}=\alpha_{0}}\right) \\
& +\mathrm{O}\left(\mathrm{e}^{-\left(2 \alpha_{0}+\mathrm{r}\right) \mathrm{T}}\right) .
\end{aligned}
$$

So, $\alpha_{0}^{R}$ is defined by the following equation:

$$
\frac{\sigma\left(\alpha_{0}^{R}+r\right)}{\left(2 \alpha_{0}^{R}+r\right)^{2}}-\left(1-e^{-r T}\right) c^{\prime}\left(\alpha_{0}^{R}\right)-e^{-r T} G\left(\alpha_{0}^{R}\right)+\mathrm{O}\left(\mathrm{e}^{-\left(2 \alpha_{0}^{\mathrm{R}}+\mathrm{r}\right) \mathrm{T}}\right)=0
$$

where

$$
G\left(\alpha_{0}^{R}\right) \equiv \frac{\sigma \alpha_{T}^{R}}{2 \alpha_{0}^{R}\left(2 \alpha_{T}^{R}+r\right)}+\left.\frac{\sigma}{2\left(2 \alpha_{T}^{R}+r\right)} \frac{d \alpha_{j T}^{R}}{d \alpha_{i 0}}\right|_{\alpha_{i 0}=\alpha_{j 0}=\alpha_{0}^{R}}>0 .
$$




\section{References}

[1] Bester, Helmut, and Emmanuel Petrakis, 1995, "Price Competition and Advertising in Duopoly", European Economic Review, 39, 1075-1088.

[2] Burke, Raymond R., and Thomas K. Srull, 1988, "Competitive Interference and Consumer Memory for Advertising", Journal of Consumer Research, 15, 55-68.

[3] Butters, Gerar R., 1977, "Equilibrium Distributions of Sales and Advertising Prices", Review of Economic Studies, 44, 465-491.

[4] Doob, Joseph L., 1953/1990, "Stochastic Processes", New York: Wiley Classics Library (1990 edition).

[5] Ebbinghaus, Hermann, 1885/1913, "Memory. A Contribution to Experimental Psychology", New York: Teachers College / Columbia University (1913 edition).

[6] Grossman, Gene M., and Carl Shapiro, 1984, "Informative Advertising with Differentiated Products", Review of Economic Studies, 51, 63-81.

[7] Jenkins, Jhon G., and Karl M. Dallenbach, 1924, "Obliviscence During Sleep and Walking", American Jpurnal of Psychology, 35, 605-612.

[8] Jewell, Robert D., and H. Rao Unnava, 2003, "When Competitive Interference Can Be Beneficial", Journal of Consumer Research, 30, 283-291.

[9] Keller, Kevin Lane, 1987, "Memory Factors in Advertising: The Effect of Advertising Retrieval Cues on Brand Evaluations", Journal of Consumer Research, 14, 316-333.

[10] Keller, Kevin Lane, 1991, "Memory and Evaluation Effects in Competitive Advertising Environments", Journal of Consumer Research, 17, 463-476.

[11] Kent, Robert J., and Chris T. Allen, 1994, "Competitive Interference Effects in Consumer Memory for Advertising: The Role of Brand Familiarity", Journal of Marketing, 58, 97105.

[12] Klemperer, Paul, 1987, "Markets for Consumer Switching Costs", The Quarterly Journal of Economics, 102, 375-394.

[13] Klemperer, Paul, 1995, "Competition when Consumers have Switching Costs: An Overview with Applications to Industrial Organization, Macroeconomics, and International Trade", The Review of Economic Studies, 62, 515-539.

[14] Kumar, Anand, and Shanker Krishnan 2004, "Memory Interference in Advertising: A Replication and Extension", Journal of Consumer Research, 17, 463-476.

[15] Krähmer, Daniel, 2004, "Advertising and Consumer Memory", mimeo, University College London.

[16] Lawler, Gregory F., 1995, "Introduction to Stochastic Processes", New York: Chapman \& Hall. 
[17] LeBlanc, Greg, 1998, "Informative Advertising Competition", Journal of Industrial Economics, 46, 63-77.

[18] Mullainathan, Sendhil, 2002, "A Memory-Based Model of Bounded Rationality", Quarterly Journal of Economics, 117, 735-774.

[19] Murnane, Kevin, and Richard M. Shiffrin, 1991, "Interference and Representation on Events in Memory", Journal of Experimental Psycholody, 17, 855-874.

[20] Solso, Robert L., 1988, "Cognitive Psychology", 2nd edition, New York: Allyn \& Bacon.

[21] Stegeman, Mark, 1991, "Advertising in Competitive Markets", American Economic Review, 81, 210-223.

[22] Tulving, Endel, 1974, "Cuedependent Forgetting", American Scientist, 62, 74-82.

[23] Tulving, Endel, and Z. Pearlston, 1966, "Availability versus Accessibility of Information in Memory for Words", Journal of Verbal Learning and Verbal Behavior, 5, 381-391.

[24] Underwood, Benton J., 1957, "Interference and Forgetting", Psychological Review, 64, 49-60.

[25] Wilson, Andrea, 2002, "Bounded Memory and Biases in Information Processing", mimeo, Princeton University.

[26] Woodworth, Robert S., 1938, "Experimental Psychology", New York: Henry Holt \& Company. 